\title{
In vivo tomographic imaging based on bioluminescence
}

\author{
Wenxiang Cong, Durairaj Kumar, Yubin Kang, Patrick \\ Sinn, Earl Nixon, et al.
}

Wenxiang Cong, Durairaj Kumar, Yubin Kang, Patrick Sinn, Earl Nixon, John Mienel, Melissa J. Suter, Lihong V. Wang, Geoffrey McLennan, Eric A. Hoffman, Ge Wang, "In vivo tomographic imaging based on bioluminescence," Proc. SPIE 5535, Developments in X-Ray Tomography IV, (26 October 2004); doi: 10.1117/12.560522

SPIE Event: Optical Science and Technology, the SPIE 49th Annual Meeting, 2004, Denver, Colorado, United States 


\title{
In vivo tomography imaging based on bioluminescence
}

\author{
Wenxiang Cong ${ }^{\mathrm{a}}$, Kumar Durairaj ${ }^{\mathrm{a}}$, Yubin Kang ${ }^{\mathrm{c}}$, Patrick Sinn ${ }^{\mathrm{c}}$, Earl Nixon ${ }^{\mathrm{b}}$, John Mienel Jr ${ }^{\mathrm{b}}$, \\ Melissa Suter ${ }^{\mathrm{c}}$, Lihong V. Wang ${ }^{\mathrm{d}}$, Geoffrey McLennan ${ }^{\mathrm{c}}$, Eric. A. Hoffman ${ }^{\mathrm{b}}$, and ${ }^{*}$ Ge Wang $^{\mathrm{a}}$, \\ ${ }^{a}$ Bioluminescence Tomography Laboratory, Department of Radiology \\ ${ }^{\mathrm{b}} \mathrm{CT} /$ Micro-CT Laboratory, Department of Radiology \\ ${ }^{\mathrm{c}}$ Department of Internal Medicine, \\ University of Iowa, \\ 200 Hawkins Drive, Iowa City, IA 52242, USA \\ dOptical imaging laboratory, Department of biomedical engineering \\ Texas A\&M University
}

\begin{abstract}
The most important task for bioluminescence imaging is to identify the emission source from the captured bioluminescent signal on the surface of a small tested animal. Quantitative information on the source location, geometry and intensity serves for in-vivo monitoring of infectious diseases, tumor growth, metastases in the small animal. In this paper, we present a point-spread function-based method for reconstructing the internal bioluminescent source from the surface light output flux signal. The method is evaluated for sensing the internal emission sources in nylon phantoms and within a live mouse. The surface bioluminescent signal is taken with a highly sensitive CCD camera. The results show the feasibility and efficiency of the proposed point-spread function-based method.
\end{abstract}

Keywords: Bioluminescence tomography, CT/micro-CT, molecular imaging, point-spread function (PSF).

\section{INTRODUCTION}

Biomedical applications of in vivo tomographic imaging have been instrumental for development of modern medicine. With the advent of imaging agents, functional and molecular imaging attracts more and more attention. The recent focus is to unfold molecular and cellular activities, promising to accelerate progress in diagnostic methods and therapeutic options. As a powerful way for molecular imaging, we utilize reporter virus vectors. As a result, the reporter gene expression can be captured noninvasively. The advancement of molecular biology has led to various sorts of reporter genes responsible for positron emission, florescent emission, bioluminescent emission, etc. These reporter genes would facilitate rapid translation from in vitro results to pre-clinical and clinical trials with the aid of various imaging methodologies such as single photon emission computed tomography, positron emission tomography, magnetic resonance imaging, and optical imaging. These image modalities are extensively used in small animals for tumor detection, drug monitoring, etc. The radionuclide-based techniques are sensitive and permit tomographic reconstruction, but they request expensive facilities. A competing approach for analysis of gene expressions is optical imaging. Even though the optical signal can be very blurry due to scattering in the tissue, bioluminescence imaging enjoys several distinct advantages. Particularly, it allows a rather high sensitivity, detecting a small number of cells instead of a mass of 3-5mm in size using PET ${ }^{1-6}$.

In the bioluminescent imaging experiment, a small animal, like a mouse, is infected by inhalation with luciferase (Adluc), and then is anesthetized and injected with luciferin. The chemical reaction between luciferase and luciferin emits photons. The bioluminescent light travels through the tissues, and is captured with a CCD camera when it goes out from the surface of the animal. The central issue of bioluminescent tomography is to reconstruct a bioluminescent source

*Corresponding author: ge-wang@uiowa.edu 
distribution inside a mouse from collected bioluminescent data on the surface of mouse. The bioluminescent photon propagation in the tissue can be described by the transport equation or the Monte Carlo model ${ }^{7-9}$. Generally, The inverse problem to identify the bioluminescent source is ill-posted, and more difficult than the related forward problem. Some priori knowledge is required. Wang, et al. discussed the uniqueness for this inverse source problems ${ }^{10}$, and pointed out the need of some priori knowledge, including the optical parameters of the tissues, the possible region for the light source and 3D anatomic structure of the region of interest, which can be obtained with medical imaging techniques like micro-CT scanning. In this work, we report a point spread function (PSF)-based model to describe the bioluminescent light transport, and reconstruct a bioluminescent source distribution in a small animal. Using this method, we reconstruct bioluminescent sources in both physical phantoms and living mice from bioluminescent data measured on the surfaces of these objects. Specifically, the bioluminescent data on the gene expression from the live mouse liver are combined with the corresponding micro CT segmentation to demonstrate the feasibility of in-vivo imaging for localization and quantification of chemi-luminescent activities within the organ. Finally, the relevant issues and further research directions are discussed.

\section{MATERIALS AND METHODS}

\subsection{Light source reconstruction method}

In a bioluminescent imaging experiment with a small animal, some biological cells, transfected with luminescent reportors such as luciferase, produce bioluminescence. The photons travel in the tissues, reach the external surface, and the surface output flux is recorded with a highly sensitive CCD camera. This light transport can be described with a linear system, i.e. the light flux density $f(r)$ at location $\boldsymbol{r}$ is

$$
f(\mathbf{r})=\int_{\Omega_{0}} s\left(\mathbf{r}_{0}\right) p\left(\mathbf{r}_{0}, \mathbf{r}\right) \mathrm{d} \mathbf{r}_{0} \quad\left(\mathbf{r}_{0} \in \Omega_{0}, \mathbf{r} \in \Omega\right)
$$

where $s\left(\mathbf{r}_{0}\right)$ is the light source density at location $\mathbf{r}_{0}$, and $p\left(\mathbf{r}_{\mathbf{0}}, \mathbf{r}\right)$ is a point spread function (PSF), which is generally spatially variant. The region $\Omega$ is the part of the small animal where the bioluminescent process takes place. The light source $s\left(\mathbf{r}_{0}\right)$ is distributed in a sub-region $\Omega_{0}\left(\Omega_{0} \subset \Omega\right)$ where the reporter genes are tagged with the cells. Bioluminescence tomography is to determine light source distribution $s\left(\mathbf{r}_{0}\right)$ in $\Omega_{0}$ from measurement of $f(\mathbf{r})$ on the surface of $\Omega, \partial \Omega$.

There are several methods to compute PSF $p\left(\mathbf{r}_{0}, \mathbf{r}\right)$. Monte Carlo method $^{8}$ is a flexible and accurate to study the photon transport in turbid tissues. In an infinite homogeneous medium, the PSF, denoted as $p_{0}\left(\mathbf{r}_{\mathbf{0}}, \mathbf{r}\right)$, depends only on

the distance $\left(r=\sqrt{\left(\mathbf{r}-\mathbf{r}_{0}\right) \cdot\left(\mathbf{r}-\mathbf{r}_{0}\right)}\right)$ between the source and detection position. In this case the PSF can be easily obtained with Monte Carlo simulation. Another choice is based on the diffusion equation. In bioluminescent imaging, photons propagate in a highly scattering tissue, and the diffusion approximation is sufficiently accurate to describe light propagation ${ }^{9}$. In a homogeneous medium, $p_{0}\left(\mathbf{r}_{\mathbf{0}}, \mathbf{r}\right)$ can be derived from the diffusion equation, as

$$
p_{0}\left(\mathbf{r}_{0}, \mathbf{r}\right)=\frac{\exp \left(-\mu_{e f f} r\right)}{4 \pi D r} \quad\left(r=\sqrt{\left(\mathbf{r}-\mathbf{r}_{0}\right) \cdot\left(\mathbf{r}-\mathbf{r}_{0}\right)}\right),
$$

where $D$ and $\mu_{e f f}$ are the diffusion and effective attenuation coefficients, respectively, defined in terms of the absorption coefficient $\mu_{a}$, scattering coefficient $\mu_{s}$ and anisotropy factor $g$,

$$
D=1 /\left(3\left((1-g) \mu_{s}+\mu_{a}\right)\right) \text { and } \mu_{e f f}=\left(3 \mu_{a}\left(\mu_{a}+\mu_{s}(1-g)\right)\right)^{1 / 2} .
$$

Since a small animal contains various types of tissues, the light propagation medium must be considered heterogeneous. Hence, we decompose the diffusion and absorption coefficients as $D(\mathbf{r})=D^{0}+\delta D(\mathbf{r}), \mu_{a}(\mathbf{r})=\mu_{a}^{0}+\delta \mu_{a}(\mathbf{r})$, where $D^{0}$ and $\mu_{a}^{0}$ are the reference values of the respective coefficients. The PSF in the heterogeneous medium can be expressed in an integral form ${ }^{9,11,12}$ :

$$
p\left(\mathbf{r}_{0}, \mathbf{r}\right)=p_{0}\left(\mathbf{r}_{0}, \mathbf{r}\right)+\int_{\Omega} p_{0}\left(\mathbf{r}_{0}, \zeta\right)\left(\nabla \cdot(\delta D(\zeta) \nabla p(\zeta, \mathbf{r}))-\delta \mu_{a}(\zeta) p(\zeta, \mathbf{r})\right) \mathrm{d} \zeta .
$$


where the Hamilton $\nabla$ operates with $\zeta$. According to the first-order perturbation theory, PSF $p\left(\mathbf{r}_{0}, \mathbf{r}\right)$ can be approximated with ${ }^{11,12}$ :

$$
p\left(\mathbf{r}_{0}, \mathbf{r}\right)=p_{0}\left(\mathbf{r}_{0}, \mathbf{r}\right)-\int_{\Omega} p_{0}\left(\mathbf{r}_{0}, \zeta\right) p_{0}(\zeta, \mathbf{r}) \delta \mu_{a}(\zeta) \mathrm{d} \zeta-\left(\nabla p_{0}\left(\mathbf{r}_{0}, \zeta\right)\right) \cdot\left(\nabla p_{0}(\zeta, \mathbf{r})\right) \delta D(\zeta) \mathrm{d} \zeta .
$$

In practice, 3-D geometric structures of the tissues in the small animal can be obtained from an X-ray CT/micro-CT scan, and the associated optical parameters $\left(\mu_{s}, \mu_{a}, g\right)$ be taken from an optical database ${ }^{20}$. Therefore, $p\left(\mathbf{r}_{0}, \mathbf{r}\right)$ in the heterogeneous animal can be computed by (3) or (4). Thus, the bioluminescence tomography problem becomes to reconstruct the light source distribution $S(\mathbf{x})$ inside the biological tissue region $\Omega_{0}$, so that the surface flux calculated via (1), with use of the accurate PSF (3) or the approximation (4), optimally matches the measured surface flux density $f^{\text {measured }}(\mathbf{x})$. The reconstruction can be expressed as a minimization procedure ${ }^{13}$ :

$$
\begin{aligned}
& \min _{U(\mathbf{r}) \geq S(\mathbf{r}) \geq 0}\left\{\int_{\Gamma}\left(f(\mathbf{r} ; S)-f^{\text {measured }}(\mathbf{r})\right)^{2} \mathrm{~d} \mathbf{r}+\lambda \eta(S)\right\} \\
& f(\mathbf{r} ; S)=\int_{\Omega_{0}} s\left(\mathbf{r}_{0}\right) p\left(\mathbf{r}_{0}, \mathbf{r}\right) \mathrm{d} \mathbf{r}_{0} \quad\left(\mathbf{r}_{0} \in \Omega_{0}, \mathbf{r} \in \partial \Omega\right)
\end{aligned} .
$$

in which $\Gamma$ is a sub-domain of the external surface $\partial \Omega, \eta$ is a stabilizing functional, and $\lambda$ is a fixed regularization factor. U represents that the unknown source density $S$ must be constrained within the physically meaningful range. In the experiment, the measured quantity is the output flux $Q(\mathbf{x})$ on $\Gamma$, which yields $f^{\text {measured }}(\mathbf{x})$ by

$$
\begin{aligned}
& f^{\text {measured }}(\mathbf{x})=-2 A_{\mathrm{n}}(\mathbf{x}) Q(\mathbf{x}) \\
& A_{\mathrm{n}}(\mathbf{x})=(1+R(\mathbf{x})) /(1-R(\mathbf{x}))
\end{aligned}
$$

in which $R$ depends on the local refractive index $\mathrm{n}(\mathbf{x})$ of the medium ${ }^{14}$.

\subsection{Phantom experiments}

Cylindrical tissue phantoms were made from a nylon material. Sizes of the specimens are $30 \mathrm{~mm}$ in diameter and $30 \mathrm{~mm}$ in height. The scattering coefficient, absorption coefficient, and anisotropy parameter for the material are $\mu_{s}=34 \mathrm{~mm}^{-1}, \mu_{a}=0.003 \mathrm{~mm}^{-1}$ and $g=0.90$, respectively. These parameters were determined by light transmission profile matching between experiment and numerical data ${ }^{8,9}$.

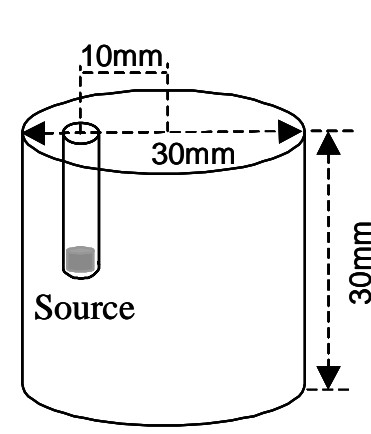

(a)

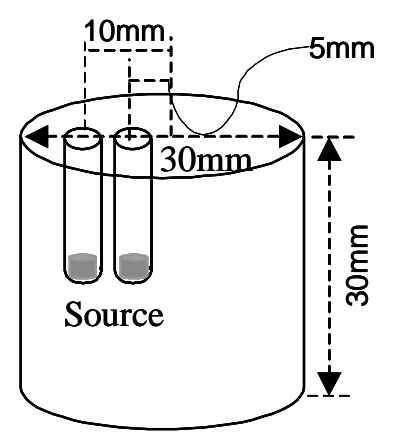

(b)

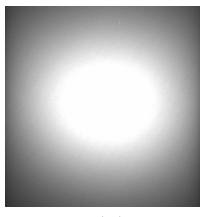

(a)

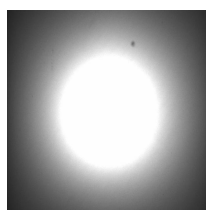

(d)

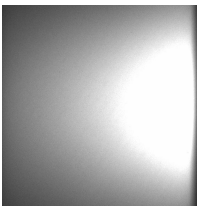

(b)

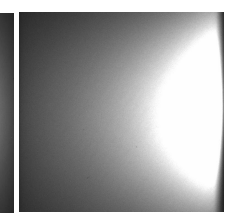

(e)

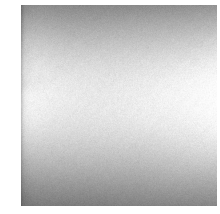

(c)

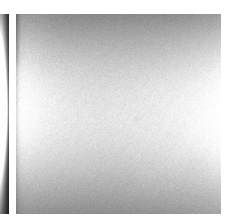

(f)

Fig 1. Bioluminescent phantoms. (a) One source phantom (distance between cylindrical axis and source mid-point: 10mm), (b) Two sources phantom (distance between cylindrical axis and source $1 \& 2$ mid-points: $5 \mathrm{~mm}$ and $10 \mathrm{~mm}$, respectively).

Fig. 2. Bioluminescent experiment for phantom. (a-c) Luminescent views for one source phantom for rotation angles of 0,90 and 180 degrees respectively; (d-f) Luminescent views for two source phantom for rotation angles of 0,90 and 180 degrees respectively. 
The phantom specimens are schematically shown in Fig. 1(a) and (b). The emission sources are small polythene tubes filled with red luminescent liquid, about $0.7 \mathrm{~mm}$ in height and $0.5 \mathrm{~mm}$ in radius. Two phantoms are prepared, one with a single source placed $10 \mathrm{~mm}$ off the axis of the specimen, the other with twin sources located at $10 \mathrm{~mm}$ and $5 \mathrm{~mm}$ off axis, respectively. The experiments were performed in a dark environment. As schematically shown in Fig. 2 (a) to 2(f), a specimen was placed on a sample holder in front of a nitrogen-cooled CCD camera, which captured the image of the output flux Q from the cylindrical surface. The exposure time was 30 seconds. The sample holder was then rotated with $90^{\circ}$, and a second bioluminescent image. Finally, the third image was taken with the holder rotated with $180^{\circ}$. Due to the symmetry, these profiles are sufficient to obtain complete light emission image on the cylindrical surface. After data acquisition, the surface output flux $\mathrm{Q}$ was first calculated by transferring the pixel grey level with the CCD image into light unit, incorporating the CCD reaction profile obtained with our meticulous calibration. Then, numerical simulations were performed.

\subsection{Mouse experiments}

In the mouse liver studies, mice were injected via the tail vein with either 50 $\mu 1$ of Ad5-CMV-Luciferase (titer: $1.8 \mathrm{x}$ $1011 \mathrm{pfu} / \mathrm{ml}$ ) or $100 \mu \mathrm{l}$ of Ad5-CMVnuclear-targeted $\beta$-galactosidase (titer: 2 x 1010pfu/ml). After three days the mice were anesthetized with i.p. injection of $2.5 \%$ avertin with a volume of $100 \mu 1 / 10 \mathrm{~g}$ body weight, Subsequently, the mice were injected i.p. with $150 \mathrm{mg} / \mathrm{kg}$ body weight of luciferin (Sigma). To detect the luciferase expression, the hair in the abdominal area was removed using hair removal lotion (Nair), and gently restrained in the imaging chamber. After luciferin administration, the mice were imaged using the CCD camera for 5 minutes. Three bioluminescent emission profiles on the surface were taken with the mouse rotated by $0^{\circ}, 90^{\circ}$ and $180^{\circ}$, respectively, which were shown in Fig. 3 (b). This three-image information reveals the transgene expression from transfected cells inside the mouse.

(a)
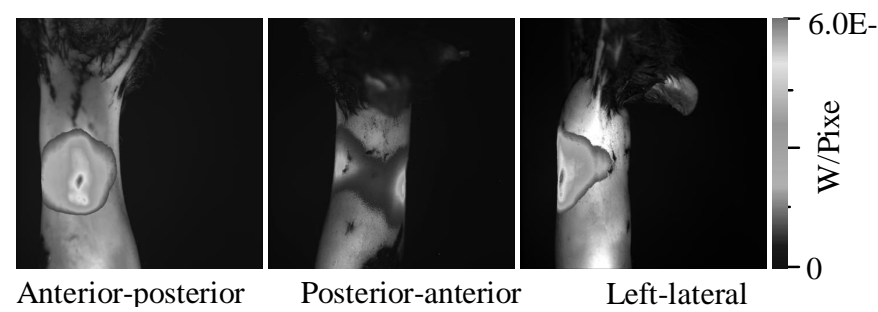

Posterior-anterior

Left-lateral
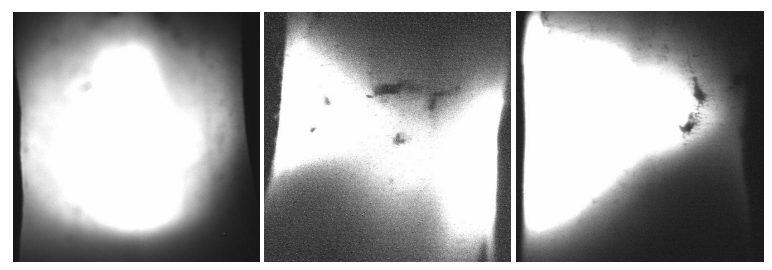

(b)

Fig. 3. In Vivo mouse liver bioluminescence tomography. In the mouse liver studies, we reconstructed a bioluminescent tail vein with either $50 \mu \mathrm{l}$ of Ad5-CMV-Luciferase (titer: $1.8 \times 1011 \mathrm{pfu} / \mathrm{ml}$ ) or $100 \mu \mathrm{l}$ of Ad5-CMV-nuclear-targeted â-galactosidase (titer: 2x 1010pfu/ml). Three days post injection, the transgene expression was detected using the CCD camera or by X-gal staining of liver tissue 5minutes after luciferin administration. It was sequentially rotated 90 degrees to acquire photographic images with lights on and bioluminescent views with lights off by exposing the camera for 0.1 seconds and 10minutes, respectively. Pseudocolor images were generated for the bioluminescent views to superpose on the corresponding regions of the photographic images (a), and bioluminescent gray level images (b). The hair in the abdominal area was removed using hair removal lotion (Nair) in the studies.

Pseudo-color images were generated for the bioluminescent views using the Adobe Photoshop software. Then, the pseudo-color images were superimposed on the corresponding regions of the photographic images of the mouse, which were shown in Fig. 3(a). After imaging, the mice were sacrificed and frozen in liquid nitrogen with the same body posture as in the bioluminescence scanning. The frozen mouse was then scanned using a Siemens Sensation 16 CT scanner and/or a SkyScan micro-CT scanner. The images of bioluminescent light emitted from the liver were anatomically recognizable from all the three angles of view. The CT image volume were segmented into major 
anatomical components including the heart, lungs, liver, stomach, bones, and so on. Then, known optical parameters (absorption coefficient $\mu_{a}$, scattering coefficient $\mu_{s}$ and anisotropy factor $g$ ) were assigned to each of components, as shown in Table $1^{14}$. A region of 250 by 400 pixels corresponds to liver part of the mouse was selected from the photographic and bioluminescent views. The pixel gray levels of a bioluminescent view were transformed into corresponding flux densities according to the intensity calibration relationship mentioned above. The transformed flux densities contained some random noise and spurious effects of various types. These data were smoothened by low-pass filtering.

Table 1. Optical parameters used in the source inversion.

\begin{tabular}{|c|c|c|c|c|}
\hline Parameter & $\mathrm{n}$ & $\mu_{a}\left(\mathrm{~mm}^{-1}\right)$ & $\mu_{s}\left(\mathrm{~mm}^{-1}\right)$ & $g$ \\
\hline Muscle & 1.37 & 0.01 & 4.0 & 0.90 \\
\hline Lung & 1.37 & 0.35 & 23.0 & 0.94 \\
\hline Bone & 1.37 & 0.002 & 20.0 & 0.90 \\
\hline Liver & 1.37 & 0.18 & 20.0 & 0.90 \\
\hline Stomach & 1.37 & 0.01 & 4.0 & 0.90 \\
\hline
\end{tabular}

\section{RESULTS}

Fig. 2 shows the luminescent emission on the surface of the tissue phantom with a single cylindrical light source. It can be observed that the brightest region is located at the middle portion of Fig. 2(a), moves to the right side of Fig. 2(b), and becomes split to both sides of Fig. 3(c) with much less brightness. The surface output flux of these three consecutive luminescent images is combined into a full surface profile as given in Fig. 4(a) and their corresponding matching counterpart is shown in Fig. 4(b) that was numerically computed by the above described PSF method. Fig. 4(c) shows the real source location whereas Fig. 4(d) depicts the source position obtained by the numerical inversion technique. The density peak of the recovered source locates exactly at the center of the real source. The total power of the emission source is found to be $3.3 \times 10^{-12}$ watts, which is a bit lower than the real value of $3.51 \times 10^{-12}$ watts by a relative error
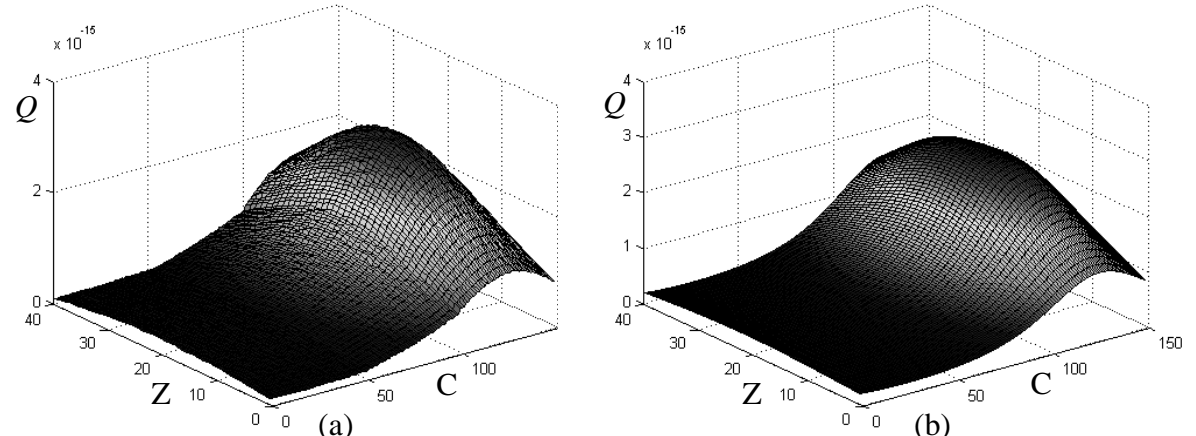

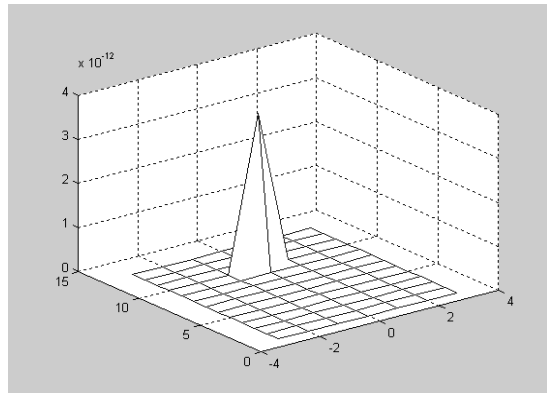

(c)

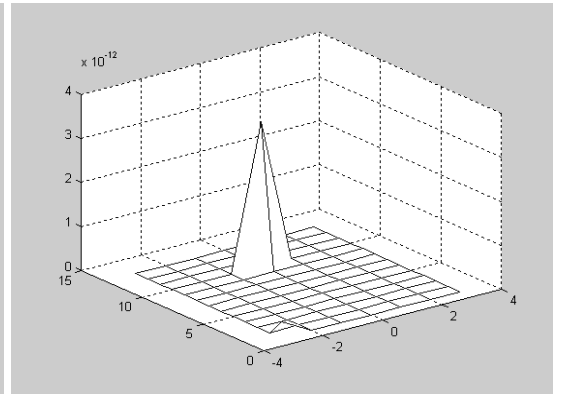

(d)

Fig. 4. Experiment with the one source phantom. (a) the experimental surface flux and (b) estimated counterpart of (a), as well as the corresponding source distributions (c) in the experiment and (d) from the reconstruction. 
Figure 5 presents the experimental and reconstruction results due to the presence of two light sources. The power is $3.5 \times 10^{-12}$ watts for both sources. The appearance of the bright region in Fig. 2(d) to 2(f) is similar to the corresponding parts in Fig. 2(a) to 2(c), however, is broader in size and is brighter. The data processing is similar to that for singlesource case. The complete surface output flux profile, Fig. 5(a), is obtained by combining Fig. 2(d) to 2(f). The real and reconstructed light source distributions are given in Fig. 5(c) and (d), respectively. The reconstruction accurately caught the center locations of the real sources. The estimated sources powers are $3.17 \times 10^{-12}$ watts $3.12 \times 10^{-12}$ watts, which are $9.4 \%$ and $10.8 \%$ lower than the real values.

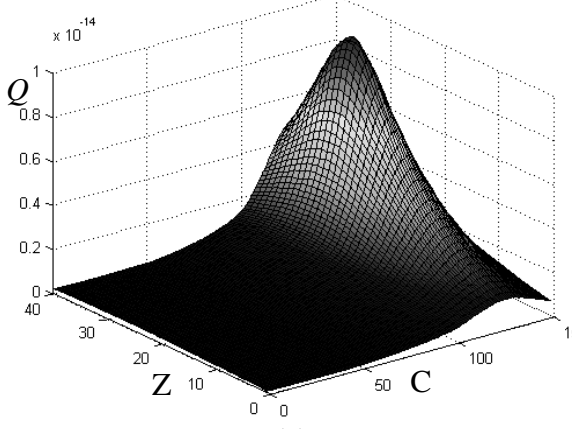

(a)

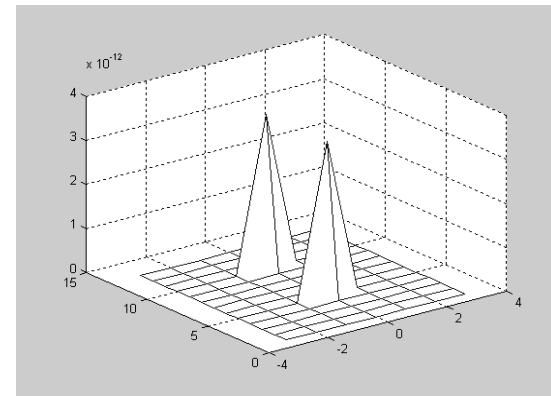

(c)

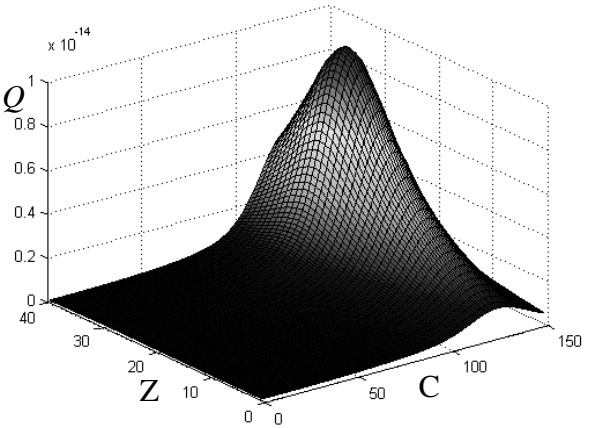

(b)

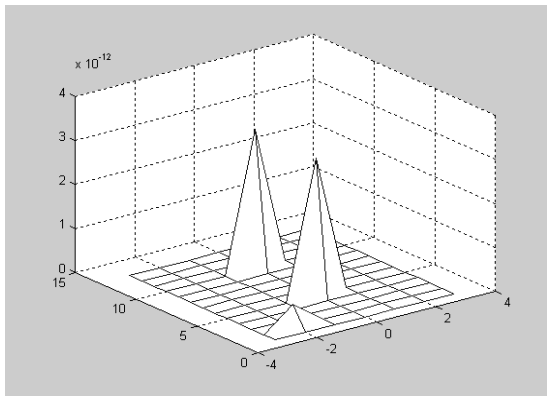

(d)

Fig. 5. Experiment with the two source phantom. (a) The experimental surface flux and (b) estimated counterpart of (a), as well as the corresponding source distributions (c) in the experiment and (d) from the reconstruction.

In vivo study results on a mouse are presented in Figures 6-7.

Three consecutive bioluminescent images obtained are shown in Fig 3(a-b), superposed on the corresponding photographs of the mouse body. The full output flux profile $Q$ around the mouse surface is obtained as Fig 6(a). The light source distribution $S$ is then reconstructed with the proposed method and is presented in Fig. 6(b). The light source is approximated as spherical sources distributed in the liver region of the mouse. The output flux corresponds to the reconstructed light source is shown in Fig. 6(c). For better comparisons, representative slices are taken from Fig. 6(a) and (c) with $\mathrm{Z}=3,8,13,18,23$ and 28, respectively, and are shown as Fig. 7(a-f), with continuous lines for the experimental data and dotted lines for the simulation results. Again, an excellent agreement was obtained, which validates the proposed reconstruction method for in vivo bioluminescent study. 

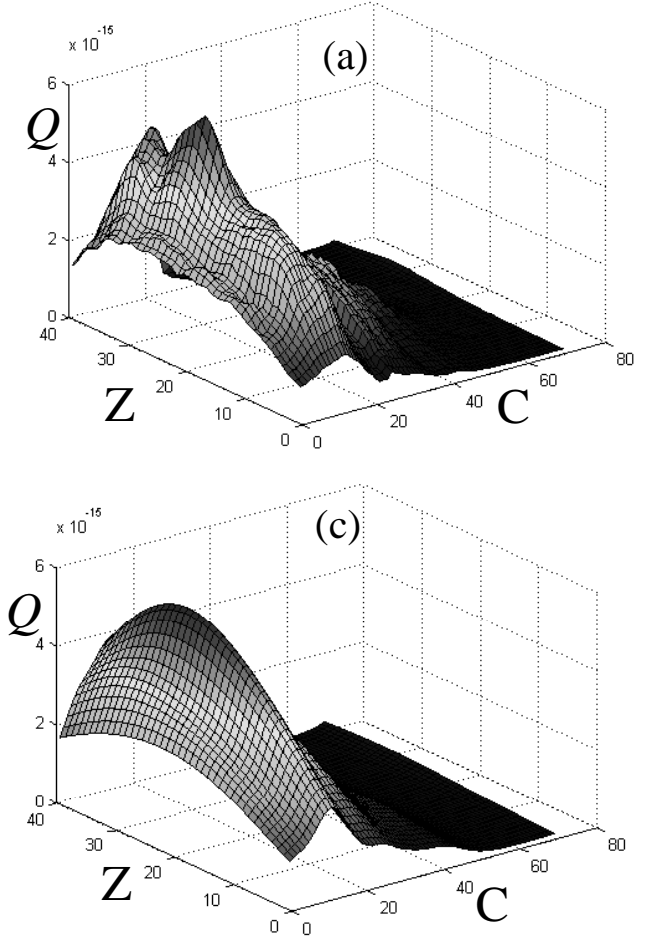

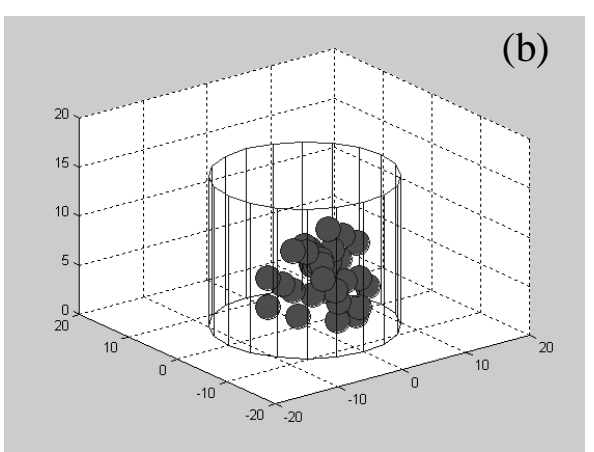

Fig. 6. (a) In vivo bioluminescent output flux $Q$ on the surface of a mouse; (b) Reconstructed light source; (c) Numerical results for $Q$ with the reconstructed light source. $\mathrm{Z}$ is with the longitudinal direction and $\mathrm{C}$ the circumference direction.

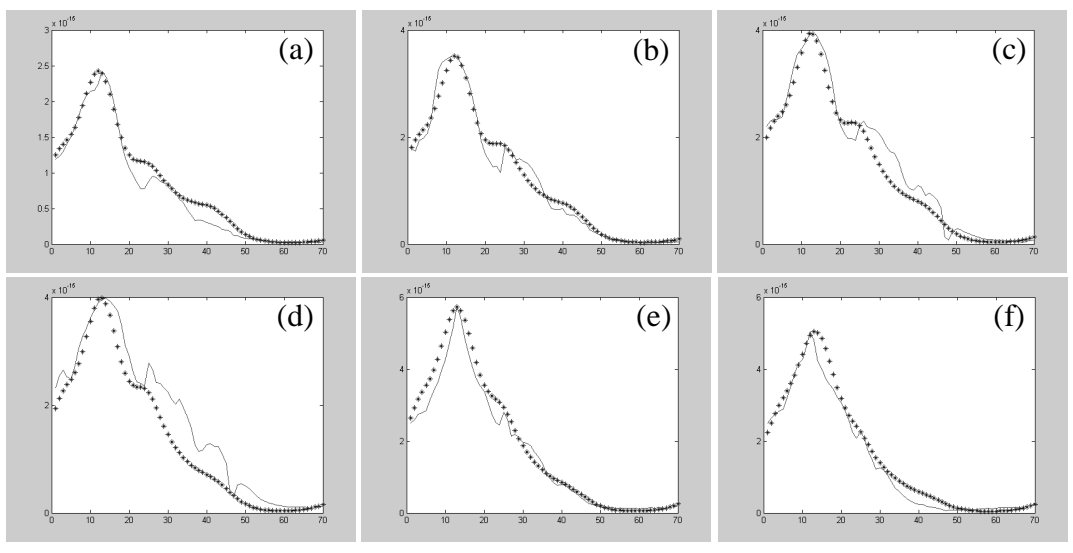

Fig. 7. Representative slices are taken from Fig. 6(a) and (c) with difference $Z$ : (a) $Z=3$; (b) $Z=8$; (c) $Z=13$; (d) $Z=18$; (e) $Z=23$ and (f) $Z=28$. Continuous lines correspond with Fig. 6(a), dotted lines with Fig. 6(c).

\section{DISCUSSIONS AND CONCLUSION}

The tissue phantoms have been fabricated with nylon material whose optical characteristics fall within the range of the biological tissues. In the luminescent imaging experiment using phantoms with one or two sources, the brightness variation in the images from different orientations is due to the proximity of the luminescent sources to the periphery of the cylinder. The diffuse light flux pattern on the surface reveals that the photons have undergone absorption and 
multiple scattering inside the medium. The experimentally measured surface output flux has been utilized to localize and quantify the sources. To solve the inverse source problem, a PSF method has been developed and applied successfully. The estimated source location information using the proposed method has well matched the true location inside the phantom. The estimated source strength variation (up to 10.8\%) can be explained by the inaccuracy in determining the optical parameters and measuring the source flux. These phantoms experimental results have indicated that the PSF method can be indeed employed for bioluminescence tomography.

Appropriate prior knowledge obtained by $\mathrm{CT} /$ micro-CT has been incorporated into the animal model to overcome the ill-poseness of the inverse problem. In the bioluminescent imaging experiments, the mouse liver has been considered. It is larger than the other organs and subject to transfect with adenovirus, which leads to enzymatic catalysis in the whole organ. Hence, the light emitting sources has been spread in the entire liver. The signal from the source undergoes multiple scattering and absorption before reaching the surface of the animal. The experiment data have shown that the bioluminescent data have provided sufficient information for quantification of the underlying light source. Further work is in progress to perform the in-vivo measurement of the optical parameters of various organs of the mouse, and to improve the reconstruction algorithm for higher accuracy and faster speed.

In conclusion, tissue phantoms have been made and used in the experiments to evaluate the PSF method for localization and quantification of an underlying bioluminescent source distribution. The preliminary results with both the phantoms and living mice have demonstrated that bioluminescence tomography is indeed doable by incorporating the prior knowledge obtained by CT/micro-CT. Further possibilities are open for bioluminescent imaging of the small animals. We are actively pursuing along this direction, and will report more results in the future.

\section{Acknowledgment}

This work is partially supported by NIH/NIBIB grants (EB001685).

\section{REFERENCES}

1. M. Rudin and R. Weissleder, "Molecular imaging in drug discovery and development", Nat. Rev. Drug Discovery, 2, 123-131, 2003.

2. S. Wang, J. Petravicz, X. O. Breakefield, "Bioluminescence Imaging of regulated gene expression, using HSV-1 Amplicon vectors in rodent brain", Molecular Therapy, 9, S27,2004.

3. Christopher H. Contag and Michael H. Bachmann, "Advances in in vivo bioluminescence imaging of gene expression”, Аnnu. Rev. Biomed. Eng., 4, 235-60, 2002.

4. G. Vassaux and T. Groot-Wassink, "In Vivo Noninvasive Imaging for Gene Therapy", J. Biomed. Biotechnol., 2, 92-101, 2003.

5. Pritha Ray, Anna M. Wu and Sanjiv S. Gambhir, "Optical Bioluminescence and Positron Emission Tomography Imaging of Novel Fusion reporter Gene in Tumor Xenografts of Living Mice", Cancer Research, 63, 1160-1165, 2003.

6. B. W. Rice, M. D. Cable and M. B. Nelson, In vivo imaging of light-emitting probes, Jl. of Biomedical Optics, 6, pp. 432-440, 2001.

7. S. R. Arridge, M. Schweiger, M. Hiraoka and d. T. Delpy, "A finite element approach for modeling photon transport in tissue", Med. Phys., 20, 299-309, 1993.

8. L-H. Wang, S. L. Jacques and L-Q Zheng, "MCML---Monte Carlo modeling of light transport in multi-layered tissues", Comput Methods and Programs in Biomed., 47, 131-146, 1995.

9. S. R. Arridge, "Optical tomography in medical imaging", Inverse problems, 15, R41-R93, 1999.

10. Ge Wang, Yi Li and Ming Jiang, "Uniqueness Theorems in Bioluminescence Tomography", Medical Physics (In press).

11. S. Economou, Green's Functions in Quantum Physics, Springer-Verlag, New York, 1983.

12. V. A. Markel and J. C. Schotland, "Inverse problems in optical diffusion tomography. I. Fourier-Laplace inversion formulas", J. Opt. Soc. Am. A., 18, 1336-1347.

13. P. P. B. Eggermont, "Maximum entropy regularization for fredholm integral equation of the first kind", SIAM J. Math. Anal., 24, 1557-1576, 1993.

14. A. J. Welch and M. J. C. van Gemert, Optical and Thermal response of laser-irradiated tissue, Plenum Press, New York, 1995. 